\title{
Enteroaggregative Escherichia coli associated with a foodborne outbreak of gastroenteritis
}

\begin{abstract}
Correspondence
Gaia Scavia

gaia.scavia@iss.it
\end{abstract}

Received 20 February 2008

Accepted 1 June 2008

\author{
Gaia Scavia, ${ }_{1}^{1}$ Monica Staffolani, ${ }^{2}$ Stefano Fisichella, ${ }^{2}$ Gianluca Striano, ${ }^{2}$ \\ Stefano Colletta, ${ }^{3}$ Giovanni Ferri, ${ }^{3}$ Martina Escher, ${ }^{1}$ Fabio Minelli ${ }^{1}$ \\ and Alfredo Caprioli ${ }^{1}$ \\ 1 Dipartimento di Sanità Pubblica Veterinaria e Sicurezza Alimentare, Istituto Superiore di Sanità, \\ Viale Regina Elena, 299, 00161 Rome, Italy \\ ${ }^{2}$ Istituto Zooprofilattico Sperimentale dell'Umbria e delle Marche, Macerata, Italy \\ ${ }^{3}$ Azienda Sanitaria Unica Regionale, Civitanova Marche, Italy
}

\begin{abstract}
This study investigated two foodborne outbreaks of gastroenteritis that occurred 10 days apart among individuals who had meals at the restaurant of a farm holiday resort. Mild gastrointestinal symptoms were reported and none of the patients needed hospitalization. Mean incubation times were 45 and $33 \mathrm{~h}$, and the overall attack rates were 43.5 and $58.3 \%$, respectively. Stool sample examination was negative for common enteric pathogens in both outbreaks. Specimens from 13 people involved in the second outbreak and 3 restaurant staff were examined for diarrhoeagenic Escherichia coli. An enteroaggregative E. coli (EAEC) strain of serotype O92: H33 was isolated from six participants and one member of staff. In particular, the EAEC strain was isolated from five of the six cases of diarrhoea examined. The strain showed an aggregative pattern of adherence to HEp-2 cells, did not produce a biofilm and possessed the virulence-related genes aat, aggR, aap and set1 $A$, but not the ast $A$ gene. A retrospective cohort study indicated a pecorino cheese made with unpasteurized sheep milk as the possible source $(P<0.001)$. Samples of the cheese had E. coli counts higher than $10^{6}$ c.f.u. $\mathrm{g}^{-1}$, but the outbreak EAEC strain was not isolated. This report confirms that EAEC infections are probably underdiagnosed because of the limited availability of laboratories capable of identifying this group of pathogenic E. coli.
\end{abstract}

\section{INTRODUCTION}

Enteroaggregative Escherichia coli (EAEC) are diarrhoeagenic $E$. coli characterized by the ability to adhere to HEp-2 cells in a characteristic 'stacked-brick' pattern (Nataro \& Kaper, 1998). This property is usually due to the presence of aggregative adherence fimbriae, whose expression is positively controlled by the $\operatorname{aggR}$ gene, located on a large plasmid termed pAA (Nataro, 2005). EAEC infections are usually associated with watery diarrhoea, which is often persistent (Nataro \& Kaper, 1998). Illness results from a complex interaction between pathogen and host, which implicates the initial adherence of the bacteria to the epithelium of terminal ileum and colon, in the characteristic aggregative pattern, followed by damage and a subsequent inflammatory response of the intestinal mucosa (Nataro, 2005; Huang et al., 2006).

Abbreviations: $95 \% \mathrm{Cl}, 95 \%$ confidence interval; EAEC, enteroaggregative Escherichia coli; ISS Istituto Superiore di Sanità; OB1/2, outbreak 1/2; VT, verocytotoxin.
EAEC are a major cause of protracted diarrhoea in children in developing countries (Nataro \& Kaper, 1998; Huang et al., 2006), where they are also linked to diarrhoeal illness of travellers and human immunodeficiency virus-positive patients (Huang et al., 2006). However, they have also been found to be associated with diarrhoea in industrialized countries (Tompkins et al., 1999; Weintraub, 2007). EAEC belong to a wide range of serotypes (Wilson et al., 2001) and are considered as emerging pathogens, characterized by a varied and complex epidemiology (Huang et al., 2006). Cases of EAEC infection are reported to be sporadic, but some outbreaks, involving both children and adults, have been described. The sources of infection have rarely been identified in these episodes. In France, an outbreak due to an atypical EAEC serogroup O111 capable of producing verocytotoxin (VT) (Morabito et al., 1998) was possibly due to person-to-person transmission (Boudailliez et al., 1997). In India, a community-wide outbreak in a village was epidemiologically associated with the consumption of water from open wells (Pai et al., 1997). Three foodborne outbreaks have been reported in Japan. The first, caused by an untypable EAEC strain, 
involved over 2500 students eating a school lunch that was epidemiologically identified as the cause of the outbreak (Itoh et al., 1997). The second and third involved highschool students, and adults attending a party, and were associated with EAEC strains belonging to serogroups O126 and O111, respectively (Yatsuyanagi et al., 2002). No information on the food items possibly involved was available. Another four foodborne outbreaks have been described in the UK (Smith et al., 1997). The settings included restaurants (two outbreaks) and dinners organized for Christmas and a conference, and the number of people involved ranged from 14 to 111. EAEC strains belonging to different serogroups were isolated from three of the outbreaks, but no information on the possible sources was available.

In this paper, we report on the investigation of two foodborne outbreaks of gastroenteritis that occurred in Italy, associated with the restaurant of a farm holiday resort. An EAEC strain of serotype O92: H33 was isolated from the patients involved in one of these outbreaks, which was specifically investigated for these pathogens.

\section{METHODS}

Unless specified, all of the activities and the definition refer to both outbreaks; the terms outbreak 1 (OB1) and outbreak 2 (OB2) refer to the clusters of cases associated with the meals of 16 and 26 February 2006, respectively.

Background On 20 February 2006, the Territorial Health Unit of Civitanova Marche, a town located in the Marche region of central Italy, was informed of several cases of gastroenteritis among patients who had attended a dinner, held 4 days before, in a restaurant located on a farm holiday resort. Epidemiological and microbiological investigations were carried out with inconclusive results, and no evidence of gross failures in the hygiene procedures and conditions was observed in the restaurant. On 3 March 2006, a new cluster of cases of gastroenteritis with symptoms similar to those reported in the previous outbreak was notified to the same Health Unit. Cases were identified among people who had participated in a birthday banquet that had taken place in the same restaurant on 26 February 2006. The activity of the restaurant was immediately suspended. As the farm owned a sheep flock and used unpasteurized raw milk to produce a typical cheese (pecorino) usually served in the restaurant, pasteurization of the milk was also ordered.

Epidemiological investigation. All of the attendees were traced back and interviewed using a standard questionnaire to collect information on age, gender, whether the participant became ill or not, type and time of onset of clinical symptoms and consumption of food items. A case of gastroenteritis was defined as a person who had attended the meal on 16 or 26 February and developed diarrhoea (three or more semi-solid or liquid stools in $24 \mathrm{~h}$ ) over the next 7 days.

Three of the restaurant staff, one of whom was also involved in cheese preparation, were also included in the OB2 investigation. They were asked to report any clinical symptoms that had occurred in the previous 2 weeks, as well as to provide a list of food items consumed by themselves on 26 February. The analytical studies were performed using a retrospective cohort design in order to identify any association between consumption of food items and cases of gastroenteritis.
Sample collection. All participants in the meals related to the outbreaks were requested to submit stool samples. Specimens were collected from 19 people involved in OB1 between 5 and 13 days after the meal, and from 14 people involved in OB2, 5 days after the meal; specimens were also obtained from three of the restaurant staff 7 days after the OB2 meal. The mean interval between onset of symptoms and collection of specimens was 3 days (range 2-10) for OB1 and 4 days (range 3-4) for OB2.

Aliquots of four food items left from the meal associated with OB2 (cake, crepes, ravioli and pecorino cheese) were collected and sent to the local Veterinary Public Health Laboratory for microbiological examinations. The OB2 investigation also included the sampling of five other pecorino cheese forms, with different seasoning periods (from 3 to 34 days), which were maintained in the restaurant store room. Ten pooled faecal samples were also collected from the sheep flock present in the farm. Each pool consisted of freshly voided faeces collected from three different animals.

Microbiological analyses. Stool specimens, kept at $4{ }^{\circ} \mathrm{C}$, were sent to the laboratory of the local hospital within $24 \mathrm{~h}$ of collection and examined by standard methods for the presence of common enteric pathogens: faeces were seeded on SS agar for Salmonella enterica and Shigella spp., on Listeria selective agar for Listeria monocytogenes, on Preston Campylobacter selective agar for Campylobacter spp. and on CIN (cefsulodin-Irgasan-novobiocin) agar for Yersinia enterocolitica. Aliquots of the specimens collected from the 13 people involved in OB2 and from the 3 restaurant employees were stored at $-20{ }^{\circ} \mathrm{C}$ until shipped to the National Institute of Health, Istituto Superiore di Sanità (ISS), to be examined for the presence of diarrhoeagenic E. coli and norovirus.

The presence of norovirus was assessed by RT-PCR, using the generic primers JV12I and JV13Y (Vinjé \& Koopmans, 1996), and the presence of free faecal VT by a Vero cell cytotoxicity assay (Caprioli et al., 1992). For the detection of pathogenic E. coli, stools were streaked onto MacConkey agar and three colony sweeps of lactose-fermenting colonies resembling $E$. coli from each plate were tested by the Vero cell assay for VT and heat-labile enterotoxin production as described previously (Bisicchia et al., 1985). Colony sweeps were also tested by PCR amplification for the presence of genes encoding VT ( $v t \times 1$ and $v t \times 2)$, intimin (eae) and the antiaggregation protein transporter gene (aat; previously reported as CVD 432) of EAEC (Schmidt et al., 1995; Nishi et al., 2003), as described previously (Morabito et al., 1998). Food samples were examined for the presence of Salmonella enterica, L. monocytogenes, Campylobacter spp. and Clostridium perfringens by standard methods. E. coli were enumerated using the EN ISO 16654:2001 procedure (ISO, 2001).

For the detection of EAEC, sheep faeces and enrichment cultures of pecorino cheese were streaked onto MacConkey agar and colony sweeps were examined for the presence of the aat gene by PCR (Morabito et al., 1998). For the isolation and characterization of EAEC strains, single colonies were picked from the aat PCR-positive colony sweeps and tested again by PCR. The aat-positive strains obtained were examined using a HEp-2 cell-adherence assay, as described previously (Morelli et al., 1994). The presence of the EAECassociated virulence genes ast $A$, encoding the enteroaggregative heatstable toxin (Savarino et al., 1993), set1A, encoding an enterotoxin first identified in Shigella flexneri 2a (Fasano et al., 1995), aggR, a plasmid-borne master virulence regulator (Dudley et al., 2006) and aap, encoding dispersin (Sheikh et al., 2002), was assessed by PCR as described by Huang et al. (2007). Detection of astA was also performed with a primer pair described by Savarino et al. (1993). EAEC strains 17-2 and 042 (Pet-negative mutant), kindly provided by J. P. Nataro (University of Maryland School of Medicine, MD, USA), 
were used as positive controls and a K12 strain as a negative control. Strains were tested with $\mathrm{O}$ antisera against the classical enteropathogenic E. coli serogroups (Statens Serum Institut, Copenhagen, Denmark) by the slide agglutination method. Serotyping was kindly performed by F. Scheutz (World Health Organization International $E$. coli and Klebsiella Centre, Statens Serum Institut, Copenhagen, Denmark). Antimicrobial susceptibility testing was performed by disc diffusion as described previously (Busani et al., 2004), using the following antibiotic discs: nalidixic acid, ampicillin, cefotaxime, ciprofloxacin, chloramphenicol, gentamicin, kanamycin, streptomycin, sulfonamides, tetracycline, trimethoprim-sulfamethoxazole. PFGE was performed as described previously (Morabito et al.,

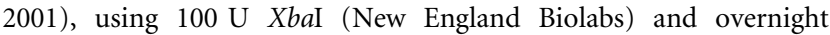
digestion. A strain of Salmonella enterica serotype Braenderup was used as a standard molecular mass marker. Biofilm formation on plastic surfaces was assessed as described by Mohamed et al. (2007). Strain 042 served as a positive control and an E. coli K12 strain as a negative control.

Data analysis. Category variables were described using counts and percentages with a $95 \%$ confidence interval $(95 \% \mathrm{CI})$. Differences in proportions were assessed for statistical significance using a $\chi^{2}$ test or, where appropriate, Fisher's exact test. For every food item, specific attack rates in exposed and unexposed individuals were calculated. The food-specific risk ratio with $95 \% \mathrm{CI}$ and $P$ values were calculated by univariate analysis with illness status as the outcome variable and consumption of each food item as the explanatory variable. Similarly, for OB2, the food-specific risk ratio was also obtained by considering the EAEC infection as the outcome variable. Statistical analyses were carried out using STATA software version 8.1 (Stata)

\section{RESULTS}

\section{Descriptive epidemiology}

Questionnaires were collected from all subjects who attended the meals associated with OB1 (23 individuals) and OB2 (24 individuals). Information on age was not available for $\mathrm{OB} 1$; the median age of the individuals involved in OB2 was 22 years (range 4-74). Symptoms included diarrhoea (10 individuals for OB1 and 14 for OB2), nausea (12 and 11 individuals, respectively), abdominal pain (10 and 11 individuals, respectively), vomiting ( 6 and 9 individuals, respectively) and hyperthermia ( 6 and 6 individuals, respectively). Symptoms were generally mild and were reported by 15 individuals involved in OB1 and 16 involved in OB2. None of the patients needed hospitalization. The mean time between meal consumption and onset of symptoms (incubation time) was $45 \pm 17 \mathrm{~h}$ for OB1 (range 11-70) and $33 \pm 16 \mathrm{~h}$ (range 19-67) for OB2. The three employees of the restaurant denied having had any symptoms in the previous 2 weeks and did not provide information on the food items consumed at the time of OB2.

A total of 10 of the 23 individuals involved in OB1 and 14 of the 24 involved in OB2 met the case definition. The overall attack rate was $43.5 \%$ in OB1 and $58.3 \%$ in OB2. The epidemic curves of OB1 and OB2 (not shown) both supported a point source exposure.

\section{Microbiological results}

In both outbreaks, all stools samples were negative for Salmonella, Shigella, Listeria, Campylobacter and Yersinia. The 16 OB2-related stool specimens sent to ISS were collected from 6 individuals with gastroenteritis, 2 individuals who had complained of symptoms other than diarrhoea (nausea and abdominal pain), 5 asymptomatic subjects and the 3 restaurant employees. All were negative for the presence of norovirus RNA and free of faecal VT. E. coli colony sweeps were obtained from all 16 samples, and were negative for VT and heat-labile enterotoxin production in the Vero cell assay. PCR analyses showed that the sweeps were negative for the $v t x 1, v t x 2$ and eae genes. Seven sweeps were positive for the aat gene, characteristic of EAEC. E. coli strains positive for the aat gene were isolated in pure culture from all seven colony sweeps. All showed an aggregative pattern of adherence when tested in the HEp-2 cell-adherence assay, and possessed the aggR, aap and set $1 A$ genes, but were negative for ast $A$. All of the isolates were negative in the biofilm production assay, with mean $\mathrm{OD}_{570}$ values ranging from 0.128 to 0.202 , compared with a mean $\mathrm{OD}_{570}$ value of 1.694 obtained with the positive-control strain 042 .

All of the strains were negative when tested with antisera against the typical enteropathogenic E. coli serogroups, were susceptible to all of the antimicrobials tested and showed similar PFGE profiles (Fig. 1). A representative strain was sent to the World Health Organization International E. coli and Klebsiella Centre for serotyping, and was identified as E. coli O92: H33.

Five of the seven EAEC strains isolated were from five of the six cases of gastroenteritis whose faeces were sent to ISS, one was from one of the five asymptomatic subjects and one was from one of the three restaurant employees; the latter was not involved in the preparation of the pecorino cheese but served as a waiter during the meal.

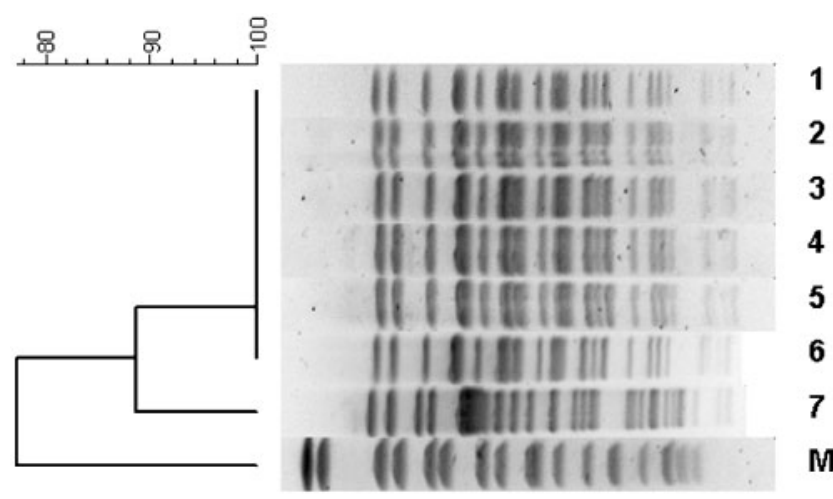

Fig. 1. PFGE patterns of representative EAEC strains isolated from individuals involved in the outbreak. The DNA was digested with Xbal. Lanes 1-6, outbreak strains; lane 7, EAEC reference strain 17-2; lane $M$, molecular mass marker (Salmonella enterica serotype Braenderup). The dendrogram was generated using the BioNumerics software package (Applied Maths) and shows the relationships among the PFGE patterns. 
All of the food specimens left from the meal associated with OB2 were negative for Salmonella, Listeria, Campylobacter and Clostridium perfringens. However, the pecorino cheese had an E. coli count greater than $10^{6}$ c.f.u. ${ }^{-1}$. The cheese specimen was kept frozen and sent to ISS to be examined for EAEC, together with samples from another five forms of pecorino cheese maintained in the restaurant store room. All of these samples were negative for the presence of EAEC, as well as the ten samples of pooled faeces collected from the farm sheep flock.

\section{Analytical epidemiology}

A total of 22 food items were served in the meal associated with OB1 and 16 in the meal associated with OB2. Twelve food items were served in both meals. In OB1, only two food items (jam and roasted potatoes) had a statistically significant risk ratio ( $P=0.029$ for both items). In OB2, a statistically significant association was observed between consumption of ham $(P=0.029)$ and pecorino cheese $(P<0.001)$, and cases of illness. The risk ratio could not be calculated for these items as no cases of illness in the unexposed subjects were recorded. Attack rates, risk ratio and $95 \% \mathrm{CI}$, and $P$ values for those items significantly associated with illness in at least one of the outbreaks are shown in Table 1. In both outbreaks, the pecorino cheese was served together with jam on the same plate.

Among the individuals involved in $\mathrm{OB} 2$ whose faecal samples were examined for EAEC, the isolation of this organism from stools was associated with the consumption of pecorino cheese (six EAEC-positive out of eight exposed compared with none of the five unexposed, $P=0.008$ ) and fried olives (six EAEC-positive out of nine exposed compared with none of the four unexposed, $P=0.026$ ).

\section{DISCUSSION}

The isolation of the same EAEC O92:H33 strain from five of the six cases of gastroenteritis whose faeces were examined for this pathogen, in the absence of other common enteric pathogens, strongly supports its possible aetiological role in OB2. Besides the aat gene, used as a diagnostic marker for EAEC detection (Schmidt et al., 1995; Nishi et al., 2003), the outbreak strain possessed other virulence-related genes that have been commonly associated with EAEC diarrhoea: aap, encoding the secreted protein dispersin (Sheikh et al., 2002); aggR, encoding a master regulator of a package of plasmid virulence genes (Nataro et al., 1994), including aap itself (Sheikh et al., 2002); and set1A, encoding Shigella enterotoxin 1 (Fasano et al., 1995). The association between aap and $\operatorname{aggR}$ has frequently been observed in EAEC (Huang et al., 2007; Samie et al., 2007) and is due to their presence within the same genetic locus (Czeczulin et al., 1999; Sheikh et al., 2002). The presence of aap and aggR has been associated with the production of biofilms on abiotic surfaces (Mohamed et al., 2007), although none of the EAEC strains isolated here was a biofilm producer. Despite the absence of the astA gene, the outbreak strain possessed all of the main virulence genes found to be associated with EAEC diarrhoea by Huang et al. (2007). Therefore, it can be considered a typical EAEC, according to the classification proposed by Nataro (2005).

EAEC strains belonging to serotype O92: $\mathrm{H} 33$ have been reported in a case of traveller diarrhoea in Mexico (Nataro et al., 1995) and in a child with diarrhoea in Brazil (Gomes et al., 1998). Similar to the strains described here, both of those isolates were $\operatorname{aggR}$ positive and astA negative (Nataro et al., 1995; Jiang et al., 2002; Uber et al. 2006). The Mexican O92: H33 strain, termed JM221 (Nataro et al., 1995), has been used for experimental infections in different studies with conflicting results. Nataro et al. (1995) reported that this strain failed to elicit diarrhoea when fed to five adult volunteers. Conversely, the same strain was reported elsewhere to cause mild diarrhoea in adult volunteers (Mathewson et al., 1986) and diarrhoea in gnotobiotic piglets (Tzipori et al., 1992). These results suggest that EAEC O92: H33 can be only moderately virulent, and this feature could explain the mildness of the symptoms observed in the patients involved in this outbreak.

Table 1. Food items statistically associated with cases of gastroenteritis in at least one of the outbreaks

Attack rate (AR) among exposed and unexposed individuals, relative risk ratio (RR) and $95 \% \mathrm{CI}$, and $P$ values are shown.

\begin{tabular}{|c|c|c|c|c|c|}
\hline \multirow[t]{2}{*}{ Outbreak } & \multirow[t]{2}{*}{ Food item } & \multicolumn{2}{|c|}{ No. of cases of gastroenteritis among: } & \multirow[t]{2}{*}{$\mathrm{RR}(95 \% \mathrm{CI})$} & \multirow[t]{2}{*}{$P$ value } \\
\hline & & Exposed (AR) & Unexposed (AR) & & \\
\hline \multirow[t]{4}{*}{ OB1 } & Ham & $8 / 20(40.0 \%)$ & $2 / 3(66.7 \%)$ & $0.6(0.2-1.5)$ & 0.385 \\
\hline & Jam & $9 / 15(60.0 \%)$ & $1 / 8(12.5 \%)$ & $4.1(1.1-20.2)$ & 0.029 \\
\hline & Pecorino cheese & $10 / 21(47.6 \%)$ & $0 / 2(0 \%)$ & Undefined & 0.194 \\
\hline & Roasted potatoes & $9 / 15(60.0 \%)$ & $1 / 8(12.5 \%)$ & $4.8(1.1-20.2)$ & 0.029 \\
\hline \multirow[t]{4}{*}{ OB2 } & Ham & $14 / 21(66.7 \%)$ & $0 / 3(0 \%)$ & Undefined & 0.029 \\
\hline & Jam & $6 / 7(85.7 \%)$ & $8 / 17=47.1 \%$ & $1.8(0.9-3.6)$ & 0.081 \\
\hline & Pecorino cheese & $14 / 18(77.8 \%)$ & $0 / 6(0 \%)$ & Undefined & $<0.001$ \\
\hline & Roasted potatoes & Not served & Not served & - & - \\
\hline
\end{tabular}


This study represents what is believed to be the first report of an outbreak of EAEC infection in Italy, and we cannot exclude that EAEC may also have played a role in the outbreak occurring in the same restaurant 10 days earlier in which the stool samples were negative for common enteric pathogens and were not investigated for EAEC infection.

EAEC are considered to be emerging enteric pathogens, and this episode may confirm the possibility that their role in diarrhoeal illnesses may have been understated, as they are not looked for in many laboratories due to the difficulties of identification. In a nationwide study carried out in England with appropriate laboratory methods (Tompkins et al., 1999), EAEC was the group of pathogenic E. coli most commonly isolated from patients with intestinal infectious diseases. Whilst many other studies conducted in European countries, including Italy (Morelli et al., 1994), have described the association between EAEC and sporadic diarrhoea (Jenkins et al., 2006; Weintraub, 2007), outbreak reports are rare. The characteristics of the episode described here were quite similar to those of other outbreaks reported in industrialized countries. It occurred in a restaurant setting, the symptoms were mild and the observed attack rates (43 and $58 \%$ ) were comparable to episodes that occurred in Japan (40\%) (Itoh et al., 1997) and in the UK (between 47 and 91\%) (Smith et al., 1997). The mean incubation periods of 45 and $33 \mathrm{~h}$ observed for the two episodes were also comparable to that of $40-50 \mathrm{~h}$ reported by Itoh et al. (1997).

The epidemiological investigation strongly indicated a pecorino cheese made with unpasteurized milk as the source of the second outbreak. The cheese was prepared in house, using milk obtained from sheep owned by the resort. Microbiological analyses conducted on aliquots left over from the outbreak meal and maintained frozen, and on other whole-cheese forms produced in the same period, did not yielded EAEC. However, the high E. coli counts observed suggest that the product had been manufactured under poor hygienic conditions. Poor hygiene is considered a main risk factor for acquiring EAEC (Huang et al., 2006). In the waterborne outbreak described by Pai et al. (1997) in India, the outbreak strain was not found in the water, but the water had high coliform counts.

The involvement of a cheese made with unpasteurized milk suggested the possibility of a zoonotic origin for the EAEC outbreak strain. A recent study conducted on large numbers of cattle, sheep and pigs at slaughter in Great Britain (Cassar et al., 2004) failed to show the presence of EAEC in the intestinal content of the animals. The tests conducted on sheep faeces in this study also yielded negative results and seem to exclude our hypothesis. However, the sampling was performed 30 days after the outbreak and about 60 days after the milk used to produce the cheese was collected. Therefore, we cannot exclude the possibility that the sheep had been transiently colonized at that time by the EAEC outbreak strain, which was no longer present at the time of faeces sampling.
The detection of the outbreak strain in one of the restaurant employees confirms the occurrence of EAEC asymptomatic carriage and raises the hypothesis that inappropriate food handling by one or more individuals colonized by EAEC could have been the cause of the outbreak. The presence of EAEC in a food handler was reported in one of the outbreaks described by Smith et al. (1997), but the role of asymptomatic carriers in the epidemiology of EAEC infections remains unclear.

In conclusion, this report confirms that sporadic cases and outbreaks of diarrhoea due to EAEC occur in European countries and are probably underdiagnosed. Underdiagnosis of EAEC may occur because many public health and healthcare workers are not familiar with EAEC as a possible cause of diarrhoeal illness, and/or because limited research facilities with trained laboratory personnel are available to perform the assays to identify this pathogen. Awareness of EAEC should increase among public health officers, and samples from outbreaks in which common enteric pathogens are not detected should be submitted to laboratories specializing in EAEC diagnosis. As for other enteric pathogens, strict compliance with good hygiene practices by food handlers represents a key stage in preventing EAEC outbreaks.

\section{ACKNOWLEDGEMENTS}

This work was partially supported by the European Community Network of Excellence Med-Vet-Net (contract no. FOOD-CT-2004506122).

\section{REFERENCES}

Bisicchia, R., Ciammarughi, R., Caprioli, A., Falbo, V. \& Ruggeri, F. M. (1985). Toxin production and haemagglutination in strains of Escherichia coli from diarrhoea in Brescia, Italy. $\mathrm{J} \mathrm{Hyg} \mathrm{(Lond)} \mathrm{95,}$ 353-361.

Boudailliez, B., Berquin, P., Mariani-Kurkdjian, P., Ilef, D., Cuvelier, B., Capek, l., Tribout, B., Bingen, E. \& Piussan, C. (1997). Possible personto-person transmission of Escherichia coli O111 - associated hemolytic uremic syndrome. Pediatr Nephrol 11, 36-39.

Busani, L., Graziani, C., Battisti, A., Franco, A., Ricci, A., Vio, D., Digiannatale, E., Paterlini, F., D'Incau, M. \& other authors (2004). Antibiotic resistance in Salmonella enterica serotypes Typhimurium, Enteritidis and Infantis from human infections, foodstuffs and farm animals in Italy. Epidemiol Infect 132, 245-251.

Caprioli, A., Luzzi, I., Rosmini, F., Pasquini, P., Cirrincione, R., Gianviti, A., Matteucci, M. C. \& Rizzoni, G. (1992). Hemolytic-uremic syndrome and verocytotoxin-producing Escherichia coli infection in Italy. J Infect Dis 166, 154-158.

Cassar, C. A., Ottaway, M., Paiba, G. A., Futter, R., Newbould, S. \& Woodward, M. J. (2004). Absence of enteroaggregative Escherichia coli in farmed animals in Great Britain. Vet Rec 154, 237-239.

Czeczulin, J. R., Whittam, T. S., Henderson, I. R., Navarro-Garcia, F. \& Nataro, J. P. (1999). Phylogenetic analysis of enteroaggregative and diffusely adherent Escherichia coli. Infect Immun 67, 2692-2699.

Dudley, E. G., Thomson, N. R., Parkhill, J., Morin, N. P. \& Nataro, J. P. (2006). Proteomic and microarray characterization of the AggR 
regulon identifies a pheU pathogenicity island in enteroaggregative Escherichia coli. Mol Microbiol 61, 1267-1282.

Fasano, A., Noriega, F. R., Maneval, D. R., Jr, Chanasongcram, S., Russell, R., Guandalini, S. \& Levine, M. M. (1995). Shigella enterotoxin 1: an enterotoxin of Shigella flexneri 2a active in rabbit small intestine in vivo and in vitro. J Clin Invest 95, 2853-2861.

Gomes, T. A., Vieira, M. A., Abe, C. M., Rodrigues, D., Griffin, P. M. \& Ramos, S. R. (1998). Adherence patterns and adherence-related DNA sequences in Escherichia coli isolates from children with and without diarrhoea in São Paulo city. J Clin Microbiol 36, 3609-3613.

Huang, D. B., Mohanty, A., DuPont, H. L., Okhuysen, P. C. \& Chiang, T. (2006). A review of an emerging enteric pathogen: enteroaggregative Escherichia coli. J Med Microbiol 55, 1303-1311.

Huang, D. B., Mohamed, J. A., Nataro, J. P., DuPont, H. L., Jiang, Z. D. \& Okhuysen, P. C. (2007). Virulence characteristics and the molecular epidemiology of enteroaggregative Escherichia coli isolates from travellers to developing countries. J Med Microbiol 56, 1386-1392.

ISO (2001). Microbiology of Food and Animal Feeding Stuffs Horizontal Method for the Detection of Escherichia coli O157. ISO 16654, 2001 (Geneva: International Organization for Standardization).

Itoh, Y., Nagano, I., Kunishima, M. \& Ezaki, T. (1997). Laboratory investigation of enteroaggregative Escherichia coli $\mathrm{O}$ untypeable: $\mathrm{H} 10$ associated with a massive outbreak of gastrointestinal illness. J Clin Microbiol 35, 2546-2550.

Jenkins, C., Tembo, M., Chart, H., Cheasty, T., Willshaw, G. A., Phillips, A. D., Tompkins, D. \& Smith, H. (2006). Detection of enteroaggregative Escherichia coli in faecal samples from patients in the community with diarrhoea. J Med Microbiol 55, 1493-1497.

Jiang, Z. D., Greenberg, D., Nataro, J. P., Steffen, R. \& DuPont, H. L. (2002). Rate of occurrence and pathogenic effect of enteroaggregative Escherichia coli virulence factors in international travelers. J Clin Microbiol 40, 4185-4190.

Mathewson, J. J., Johnson, P. C., DuPont, H. L., Satterwhite, T. K. \& Winsor, D. K. (1986). Pathogenicity of enteroadherent Escherichia coli in adult volunteers. J Infect Dis 154, 524-527.

Mohamed, J. A., Huang, D. B., Jiang, Z. D., DuPont, H. L., Nataro, J. P., Belkind-Gerson, J. \& Okhuysen, P. C. (2007). Association of putative enteroaggregative Escherichia coli virulence genes and biofilm production in isolates from travelers to developing countries. J Clin Microbiol 45, 121-126.

Morabito, S., Karch, H., Mariani-Kurkdjian, P., Schmidt, H., Minelli, F., Bingen, E. \& Caprioli, A. (1998). Enteroaggregative, Shiga toxinproducing Escherichia coli $\mathrm{O} 111: \mathrm{H} 2$ associated with an outbreak of hemolytic-uremic syndrome. J Clin Microbiol 36, 840-842.

Morabito, S., Dell'Omo, G., Agrimi, U., Schmidt, H., Karch, H., Cheasty, T. \& Caprioli, A. (2001). Detection and characterization of Shiga toxin-producing Escherichia coli in feral pigeons. Vet Microbiol 82, 275-283.

Morelli, R., Baldassarri, L., Falbo, V., Donelli, G. \& Caprioli, A. (1994). Detection of enteroadherent Escherichia coli associated with diarrhoea in Italy. J Med Microbiol 41, 399-404.

Nataro, J. P. (2005). Enteroaggregative Escherichia coli pathogenesis. Curr Opin Gastroenterol 21, 4-8.

Nataro, J. P. \& Kaper, J. B. (1998). Diarrheagenic Escherichia coli. Clin Microbiol Rev 11, 142-201.

Nataro, J. P., Yikang, D., Yingkang, D. \& Walker, K. (1994). AggR, a transcriptional activator of aggregative adherence fimbria I expression in enteroaggregative Escherichia coli. J Bacteriol 176, 4691-4699.
Nataro, J. P., Deng, Y., Cookson, S., Cravioto, A., Savarino, S. J., Guers, L. D., Levine, M. M. \& Tacket, C. O. (1995). Heterogeneity of enteroaggregative Escherichia coli virulence demonstrated in volunteers. J Infect Dis 171, 465-468.

Nishi, J., Sheikh, J., Mizuguchi, K., Luisi, B., Burland, V., Boutin, A., Rose, D. J., Blattner, F. R. \& Cataro, J. P. (2003). The export of coat protein from enteroaggregative Escherichia coli by a specific ATPbinding cassette transporter system. J Biol Chem 278, 45680-45689.

Pai, M., Kang, G., Ramakrishna, B. S., Venkataraman, A. \& Muliyil, J. (1997). An epidemic of diarrhoea in South India caused by enteroaggregative Escherichia coli. Indian J Med Res 106, 7-12.

Samie, A., Obi, C. L., Dillingham, R., Pinkerton, R. C. \& Guerrant, R. L. (2007). Enteroaggregative Escherichia coli in Venda, South Africa: distribution of virulence-related genes by multiplex polymerase chain reaction in stool samples of human immunodeficiency virus (HIV)positive and HIV-negative individuals and primary school children. Am J Trop Med Hyg 77, 142-150.

Savarino, S. J., Fasano, A., Watson, J., Martin, B. M., Levine, M. M., Guandalini, S. \& Guerry, P. (1993). Enteroaggregative Escherichia coli heat-stable enterotoxin 1 represents another subfamily of $E$. coli heatstable toxin. Proc Natl Acad Sci U S A 90, 3093-3097.

Schmidt, H., Knop, C., Franke, S., Aleksic, S., Heesemann, J. \& Karch, H. (1995). Development of PCR for screening of enteroaggregative Escherichia coli. J Clin Microbiol 33, 701-705.

Sheikh, J., Czeczulin, J. R., Harrington, S., Hicks, S., Henderson, I. R., Le Bouguénec, C., Gounon, P., Phillips, A. \& Nataro, J. P. (2002). A novel dispersin protein in enteroaggregative Escherichia coli. J Clin Invest 110, 1329-1337.

Smith, H. R., Cheasty, T. \& Rowe, B. (1997). Enteroaggregative Escherichia coli and outbreaks of gastroenteritis in UK. Lancet 350, 814-815.

Tompkins, D. S., Hudson, M. J., Smith, H. R., Eglin, R. P., Wheeler, J. G., Brett, M. M., Owen, R. J., Brazier, J. S., Cumberland, P. \& other authors (1999). A study of infectious intestinal disease in England: microbiological findings in cases and controls. Commun Dis Public Health 2, 108-113.

Tzipori, S., Montanaro, J., Robins-Browne, R. M., Vial, P., Gibson, R. \& Levine, M. M. (1992). Studies with enteroaggregative Escherichia coli in the gnotobiotic piglet gastroenteritis model. Infect Immun 60, 5302-5306.

Uber, A. P., Trabulsi, L. R., Irino, K., Beutin, L., Ghilardi, A. C., Gomes, T. A., Liberatore, A. M., de Castro, A. F. \& Elias, W. P. (2006). Enteroaggregative Escherichia coli from humans and animals differ in major phenotypical traits and virulence genes. FEMS Microbiol Lett 256, 251-257.

Vinjé, J. \& Koopmans, M. P. G. (1996). Molecular detection and epidemiology of small round-structured viruses in outbreaks of gastroenteritis in the Netherlands. J Infect Dis 174, 610-615.

Weintraub, A. (2007). Enteroaggregative Escherichia coli: epidemiology, virulence and detection. J Med Microbiol 56, 4-8.

Wilson, A., Evans, J., Chart, H., Cheasty, T., Wheeler, J. G., Tompkins, D. \& Smith, H. R. (2001). Characterisation of strains of enteroaggregative Escherichia coli isolated during the infectious intestinal disease study in England. Eur J Epidemiol 17, 1125-1130.

Yatsuyanagi, J., Saito, S., Sato, H., Miyajima, Y., Amano, K. \& Enomoto, K. (2002). Characterization of enteropathogenic and enteroaggregative Escherichia coli isolated from diarrheal outbreaks. J Clin Microbiol 40, 294-297. 